\title{
Audio Computer-Assisted Self Interview Compared to Traditional Interview in an HIV-Related Behavioral Survey in Vietnam
}

\author{
Linh Cu Le MD MS PhD and Lan T.H. Vu MD PhD
}

\begin{abstract}
INTRODUCTION Globally, population surveys on HIVIAIDS and other sensitive topics have been using audio computer-assisted self interview for many years. This interview technique, however, is still new to Vietnam and little is known about its application and impact in general population surveys. One plausible hypothesis is that residents of Vietnam interviewed using this technique may provide a higher response rate and be more willing to reveal their true behaviors than if interviewed with traditional methods.
\end{abstract}

OBJECTIVE This study aims to compare audio computer-assisted self interview with traditional face-to-face personal interview and selfadministered interview with regard to rates of refusal and affirmative responses to questions on sensitive topics related to HIVIAIDS.

METHODS In June 2010, a randomized study was conducted in three cities (Ha Noi, Da Nan and Can Tho), using a sample of 4049 residents aged 15 to 49 years. Respondents were randomly assigned to one of three interviewing methods: audio computer-assisted self interview, personal face-to-face interview, and self-administered paper interview. Instead of providing answers directly to interviewer questions as with traditional methods, audio computer-assisted self-interview respondents read the questions displayed on a laptop screen, while listening to the questions through audio headphones, then entered responses using a laptop keyboard. A MySQL database was used for data management and SPSS statistical package version 18 used

\section{INTRODUCTION}

The AIDS epidemic has drawn attention worldwide and has been a national concern in Vietnam. The epidemic in Vietnam is classified as a concentrated epidemic with high prevalence among defined high-risk groups, mainly injection drug users, with lower prevalence in the general population. HIV-positive cases have been reported in all 63 Vietnamese cities. As of December 31, 2009, there were 160,019 reported HIV cases and 44,050 deaths due to AIDS-related illnesses. In 2009 alone, there were 15,713 newly reported HIV cases and 2010 AIDS-related deaths.[1] Recent projections showed that by 2012 , approximately 280,000 people would be living with HIV, but this figure may still be underestimated due to the sensitive nature of the condition and potential limitations of the national HIV case reporting system. [1] In order to implement effective intervention activities, Vietnam needs accurate data on HIVIAIDS infections and high-risk behaviors in the population.

Until now, several routine surveys and surveillance systems have been used in Vietnam to collect data on high-risk behaviors, mostly carried out in high-risk groups.[2,3] Since high-risk behavior is a sensitive topic, data are subject to recall bias as well as intentional under-reporting by respondents. Audio computer-assisted self interview (ACASI) has received attention as a method for collecting sensitive information among youth in developed-country settings such as the USA, [4] as well as in developing countries in Africa and elsewhere.[5-7] Instead of providing answers directly to interviewer questions, respondents read questions displayed for data analysis with bivariate and multivariate statistical techniques. Rates of high risk behaviors and mean values of continuous variables were compared for the three data collection methods.

RESULTS Audio computer-assisted self interview showed advantages over comparison techniques, achieving lower refusal rates and reporting higher prevalence of some sensitive and risk behaviors (perhaps indication of more truthful answers). Premarital sex was reported by $20.4 \%$ in the audio computer-assisted self-interview survey group, versus $11.4 \%$ in the face-to-face group and $11.1 \%$ in the self-administered paper questionnaire group. The pattern was consistent for both male and female respondents and in both urban and rural settings. Men in the audio computer-assisted self-interview group also reported higher levels of high-risk sexual behavior-such as sex with sex workers and a higher average number of sexual partners - than did women in the same group. Importantly, item refusal rates on sensitive topics tended to be lower with audio computer-assisted self interview than with the other two methods.

CONCLUSIONS Combined with existing data from other countries and previous studies in Vietnam, these findings suggest that researchers should consider using audio computer-assisted self interview for future studies of sensitive and stigmatized topics, especially for men.

KEYWORDS Behavioral research, community surveys, public health surveillance/methods, survey methods, effect modifier, epidemiologic biases, social desirability, HIVIAIDS, Vietnam

on a laptop screen while listening to the same question through audio headphones. Respondents enter responses on an external mini keypad or regular laptop keyboard, without presence of interviewers. ACASI has been well received in many countries and is considered to provide more reliable information than other data collection methods, particularly in HIV-related studies.[5-8]

In developing-country pilots, ACASI has shown good results, $[5,8]$ with authors finding that it reports sensitive behaviors with better confidentiality and higher accuracy and also facilitates data management.[9] In recent years, the technique has been applied in Vietnam to study adolescent and youth health;[10] Le and Blum found that ACASI showed advantages with regard to respondent attitudes and perceptions of sensitive topics in such research. This work revealed higher prevalence rates for sensitive and stigmatized behaviors (such as unmarried youth having multiple sexual partners and having sex with sex workers) than did traditional survey methods.[10.11] However, ACASI has not been used in studies of Vietnam's adult population.

The US President's Emergency Plan for AIDS Relief (PEPFAR) supported MACRO International to partner with the Vietnam General Statistical Office and National Institute of Hygiene and Epidemiology to conduct the AIDS Indicator Survey in Vietnam starting in 2005. This household-based survey of the general population aged 15 to 49 years generated important information on knowledge, attitudes and practices related to HIV risk. [3] However, traditional face-to-face interview methods may 
have resulted in under-reporting of risk behaviors. Since then, no advanced techniques for sensitive data collection have been applied in Vietnam in the general population. Thus, it would be essential to conduct a pilot community-based survey of the population aged 15 to 49 years to field test ACASI, given the previous preliminary experiences of its application in young people mentioned above.

It should be noted that ACASI is not the only technique able to facilitate confidentiality and help get more valid results on sensitive topics. The randomized response technique (RRT) proposed by Warner[12] has been tested and validated and its use is well established.[13-16] However, its applicability has not been tested in Vietnam. Moreover, its application would have required significant extra preparation, time and effort on the part of the interview team to explain the process to interviewees, and data analysis and interpretation would not be as straightforward as with ACASI. Finally, a recent meta-analysis found no advantage of RRT over ACASI.[15] Hence, we did not consider piloting RRT or a combination of RRT and ACASI, which would have made the research unnecessarily complicated.

This study aimed to compare ACASI with traditional face-to-face personal interview $(\mathrm{PI})$ and self-administered paper-and-pencil interview (SA) with regard to effectiveness of data collection, affirmative response rates on sensitive questions, item refusal rates and survey refusal rates.

\section{METHODS}

Study design and sample This was a cross-sectional community pilot survey. Three cities with rural districts within their jurisdiction were selected in different geographic regions of Vietnam: Ha Noi (capital city in the Red River Delta in the north), Da Nang (coastal city in central Vietnam) and Can Tho (in the Mekong River Delta, southern Vietnam). Individuals aged 15 to 49 years by June 1, 2010 in selected households were randomized into three groups: face-to-face personal interview administration (PI), pencil-and-paper self-administered interview (SA), and ACASI. Survey sample design satisfied basic requirements for comparability among the three data collection methods and disaggregation by rural and urban location.

Multistage cluster sampling was used. We assumed there could be differences between men and women in willingness to report sensitive information; thus, each interview-method group included both sexes, making six groups in total for analysis. Sample size was calculated based on the two-sided test of comparison between groups and the following parameters: significance of $95 \%$; power of $90 \% ; p_{1}=0.05$ (based on prevalence of youth in previous studies who exhibited risk behaviors regarding injection drug use and sexual habits). The difference to be detected was 10 percentage points (it is hypothesized that if $p_{1}=0.05$, then $p_{2}$, the prevalence found in another interview method, would be 0.15); design effect $=1.2$ (based on a previous study of this type in Vietnam);[10] allowing for 5\% nonresponse. With these parameters, the anticipated sample for each cluster was 770 , for a total of 4620 persons aged 15 to 49 years in 6 clusters (male and female for each survey method arm).

With the above estimated sample size in the three cities, two districts were randomly selected in each (one urban and one rural), with a total of 770 study participants in each district. Based on
Vietnam's 2009 census data, in order to reach 770 subjects, we randomly selected 330 households in each district: of those, 110 households would be interviewed with ACASI, 110 with SA and 110 with PI. Three communes (subunits of districts) were selected in each district. Households selected by health care workers were required to have at least one resident aged 15 to 49 . The final list was entered into a computer program to generate a random code for assigning mode of interview, this information provided to supervisors and data surveyors for each group.

Instrument design and data collection To provide quality estimates for indicators in a manner that would facilitate international comparison, data were collected through two types of questionnaire: a household questionnaire and an individual questionnaire, used for both female and male respondents. The individual questionnaire included the following main sections: a) personal background; b) reproduction and pregnancy; c) marriage and sexual activities; d) attitudes/social norms with regard to HIVIAIDS; e) awareness and knowledge of sexually transmitted infections. The questionnaires were adapted from the 2006 Vietnam Population and AIDS Indicator Survey[3] and a previous survey of young people in Vietnam,[10] transferred to a database and installed on laptop computers. The questionnaires were also voice recorded and integrated into the ACASI interview software, which is based on Net programming language, using an MS SQL database. Estimated survey completion time was 45 minutes. The usual demographic profile and household/living conditions questions were asked in all households face-to-face, then the individual questionnaire was administered in one of three randomly-assigned interview modes. For each of the three data collection methods, interviewers/data collectors were matched by sex with study participants. All interviewers were aged 20 to 30 years; they received interviewing skills training and participated in a small-scale field pilot prior to starting data collection.

Ethical procedures All data were collected in the respondent's home after written informed consent. Parental consent was obtained for those aged $<18$ years at time of data collection. No name, personal identification or identifying characteristics were recorded on individual survey forms at any time. Each household form was assigned a unique ID to permit later merging with individual forms from that household and no link could be made between household background and personal identity. All information remained confidential, following standard procedures to avoid identification of households or individuals during analysis. The study protocol was approved by the Hanoi School of Public Health ethics committee and the Institutional Review Board at the US Centers for Disease Control and Prevention.

Data analysis and interpretation Face-to-face interview questionnaire and self-administered paper-and-pencil questionnaire data were merged with ACASI into a single data set. Household characteristics information collected in all three groups using the same face-to-face interview sheet was also entered and merged with individual data. All data were managed in a MySQL database with SPSS 18 for complex survey analysis. Descriptive analysis and bivariate statistics were applied, followed by logistic regression analysis. Factor analysis was used to construct measurement scales and for coding household economic level as categorized in quintiles for multinomial logistic regression and in tertiles for univariate analysis. 
Rates of respondents reporting high-risk behaviors were compared among the three data-collection methods. For categorical variables, we used the chi-square statistic to test for significance of proportions. ANOVA was used to compare number of sexual partners reported with the three interview methods. Based on previous experience in Vietnam, $[10,11]$ we anticipated greater differences in responses to sensitive topics across interview modes in men, especially younger men, than in women. Therefore, we hypothesized that there would be no significant difference in response rates or affirmative answers to non-sensitive questions across the three interview methods, and that response rates and affirmative answers to more sensitive questions related to sexual attitudes and behaviors would increase with use of less personal and more confidential data collection methods. Specifically, affirmative rates would be lower in $\mathrm{PI}$ and SA groups and highest with ACASI.

For sensitive questions such as those related to risk behaviors, we compared not only rates of reported behavior, but also rates of question refusal. Finally, multinominal logistic regression was applied to compare probability of answering "Yes" or refusing to answer (response options "No answer" and "Don't know") versus answering "No" for the question "Have you ever had sex with a sex worker?" adjusted for all important respondent characteristics: age, marital status, rural/urban location, socioeconomic status and city. This analysis was done among male subjects only, because women having sex with a sex worker is very rare in Vietnam.

\section{RESULTS}

Sample comparability The final successfully-interviewed sample consisted of 4049 individuals, $87.6 \%$ of the desired sample of $4620 ; 273(5.9 \%)$ individuals selected were unreachable at time of interviewer visit and $300(6.5 \%)$ refused to participate. The rate of successful interview was $85.5 \%$ for ACASI, $88.2 \%$ for SA and $89.2 \%$ for $\mathrm{PI}$. There were nonsignificant differences in percentages of unreachable individuals and refusals among the three data-collection methods, with slightly higher percentages of both for ACASI (Table 1).

Table 1: Interview success rate

\begin{tabular}{|l|r|r|r|r|}
\hline Characteristics & \multicolumn{4}{|c|}{ Data Collection Method } \\
\hline & PI & SA & ACASI & AII \\
\hline Total sample selected (N) & 1540 & 1540 & 1540 & 4620 \\
\hline Successful interview & 1374 & 1359 & 1316 & 4049 \\
(n, \%) & $(89.2 \%)$ & $(88.2 \%)$ & $(85.5 \%)$ & $(87.6 \%)$ \\
Unreachable sample & 80 & 81 & 110 & 271 \\
\hline (n, \%) & $(5.2 \%)$ & $(5.3 \%)$ & $(7.1 \%)$ & $(5.9 \%)$ \\
\hline $\begin{array}{l}\text { Refusal } \\
\text { (n, \%) }\end{array}$ & 86 & 100 & 114 & 300 \\
& $(5.6 \%)$ & $(6.5 \%)$ & $(7.4 \%)$ & $(6.5 \%)$
\end{tabular}

PI: Personal interview SA: Self-administered paper-and-pencil interview ACASI: Audio computer-assisted self interview

The three randomized groups were comparable in distribution of all attributes except sex; the proportion of women was slightly higher in the ACASI group (57.4\%) than in the other two (PI $53.4 \%$, SA $54.1 \%, p<0.05)$. There were no significant differences among groups for distribution of respondents' mean age, city of residence, urban or rural locality, age group, marital status, household economic status or educational level. Mean age was 30.1 years and most participants were married (62.5\%) (Table 2).
Table 2: Respondent characteristics

\begin{tabular}{|c|c|c|c|c|}
\hline \multirow[t]{2}{*}{ Characteristics } & \multicolumn{4}{|c|}{ Data Collection Method } \\
\hline & 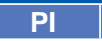 & SA & ACASI & All \\
\hline Total sample interviewed (N) & 1374 & 1359 & 1316 & 4049 \\
\hline Mean age (years) & 30.3 & 30.2 & 29.6 & 30.1 \\
\hline \multicolumn{5}{|l|}{ Cities } \\
\hline Ha Noi $(n=1304)$ & $31.1 \%$ & $32.4 \%$ & $33.1 \%$ & $32.2 \%$ \\
\hline Da Nang ( $n=1378)$ & $34.4 \%$ & $33.8 \%$ & $33.9 \%$ & $34.0 \%$ \\
\hline Can Tho $(n=1367)$ & $34.5 \%$ & $33.8 \%$ & $33.0 \%$ & $33.8 \%$ \\
\hline \multicolumn{5}{|l|}{ Location } \\
\hline Urban $(n=2003)$ & $31.1 \%$ & $32.4 \%$ & $33.1 \%$ & $32.2 \%$ \\
\hline Rural (n=2046) & $34.4 \%$ & $33.8 \%$ & $33.9 \%$ & $34.0 \%$ \\
\hline \multicolumn{5}{|l|}{ Age group } \\
\hline $15-19$ years $(n=912)$ & $21.1 \%$ & $21.9 \%$ & $24.7 \%$ & 22.5 \\
\hline $20-29$ years $(n=1073)$ & $26.2 \%$ & $26.3 \%$ & $27.1 \%$ & 26.5 \\
\hline $30-39$ years $(n=1012)$ & $25.5 \%$ & $24.4 \%$ & $25.1 \%$ & 25.0 \\
\hline $40-49$ years $(n=1052)$ & $26.5 \%$ & $26.3 \%$ & $23.2 \%$ & 25.3 \\
\hline \multicolumn{5}{|l|}{$\operatorname{Sex}^{\mathrm{a}}$} \\
\hline Male $(n=1804)$ & $45.9 \%$ & $45.1 \%$ & $42.6 \%$ & $44.6 \%$ \\
\hline Female $(n=2224)$ & $53.4 \%$ & $54.1 \%$ & $57.4 \%$ & $54.9 \%$ \\
\hline No information $(n=21)$ & $0.7 \%$ & $0.8 \%$ & $0.0 \%$ & $0.4 \%$ \\
\hline \multicolumn{5}{|l|}{ Marital status } \\
\hline Married (n=2508) & $63.9 \%$ & $63.1 \%$ & $60.3 \%$ & $62.5 \%$ \\
\hline Unmarried $(n=1541)$ & $36.1 \%$ & $36.9 \%$ & $39.7 \%$ & $37.5 \%$ \\
\hline \multicolumn{5}{|l|}{ Household SES ${ }^{b}$} \\
\hline Low $(n=1526)$ & $35.6 \%$ & $32.8 \%$ & $37.3 \%$ & $37.7 \%$ \\
\hline Average $(n=1518)$ & $39.8 \%$ & $38.8 \%$ & $40.7 \%$ & $37.5 \%$ \\
\hline High $(n=622)$ & $16.1 \%$ & $19.7 \%$ & $15.1 \%$ & $15.4 \%$ \\
\hline No information $(n=383)$ & $8.5 \%$ & $8.7 \%$ & $6.9 \%$ & $9.5 \%$ \\
\hline \multicolumn{5}{|l|}{ Educational level completed } \\
\hline Less than high school $(n=1639)$ & $42.3 \%$ & $40.0 \%$ & $39.1 \%$ & $40.5 \%$ \\
\hline High school $(n=1280)$ & $30.9 \%$ & $30.2 \%$ & $33.8 \%$ & $31.6 \%$ \\
\hline College and higher $(n=1044)$ & $24.7 \%$ & $25.9 \%$ & $26.8 \%$ & $25.8 \%$ \\
\hline No information $(n=86)$ & $2.2 \%$ & $3.8 \%$ & $0.3 \%$ & $2.1 \%$ \\
\hline
\end{tabular}

PI: Personal interview SA: Self-administered paper-and-pencil interview ACASI: Audio computer-assisted self interview

${ }^{a} p<0.05$ for ACASI vs. other two methods

${ }^{b}$ Household socioeconomic status (SES) score was developed based on main valuable household assets; the final scale was tested for reliability (Cronbach's Alpha $=0.81$ ), then categorized into tertiles.

Comparison of responses to questions of differing sensitivity Table 3 displays selected questions of differing sensitivity: two non-sensitive questions and three more sensitive questions regarding sexual and risk behaviors: premarital sex, sex with a sex worker or injection drug use. Affirmative-answer and refusalrate patterns were very similar across the three study groups for the non-sensitive questions. There was a signficant difference between the ACASI group and the other two groups in affirmative responses to two sensitive questions on premarital sex (ACASI $20.4 \%$ versus SA $11.1 \%$, PI $11.4 \%$ ) and drug use (ACASI $2 \%$ versus SA $0.5 \%$, PI 0.4\%). ACASI refusal rate for the question about premarital sex was also significantly lower than those of either SA or PI (3.4 \% versus $10.4 \%$ and $8 \%$, respectively). Data are presented for both sexes combined, since there were no significant differences between them (Table 3).

Men and women responded similarly to non-sensitive questions but men had higher affirmative response rates than women to questions about premarital sex and sex with recent casual partners. In men, the ACASI group had the highest percentage of affirmative responses to the question about premarital sex (29\% versus $14 \%$ in SA and $17.3 \%$ in $\mathrm{PI}$ ). In women, a similar pattern was observed, but at lower rates $(14 \%$ in ACASI, versus $8.7 \%$ in 
Table 3: Comparison of responses ${ }^{\mathrm{a}}$ to selected questions of differing sensitivity

\begin{tabular}{|c|c|c|c|c|c|c|}
\hline \multirow{3}{*}{ Question } & \multicolumn{6}{|c|}{ Response } \\
\hline & \multicolumn{3}{|c|}{ Yes (\%) } & \multicolumn{3}{|c|}{ Refusal (\%) } \\
\hline & $\begin{array}{c}\text { ACASI } \\
(\mathrm{N}=1540)\end{array}$ & $\begin{array}{c}\text { SA } \\
(1540)\end{array}$ & $\begin{array}{c}\mathrm{Pl} \\
(1540)\end{array}$ & $\begin{array}{l}\text { ACASI } \\
(1540)\end{array}$ & $\begin{array}{c}\text { SA } \\
(1540)\end{array}$ & $\begin{array}{c}\mathrm{Pl} \\
(1540)\end{array}$ \\
\hline $\begin{array}{l}\text { Watch TV } \\
\text { almost daily }\end{array}$ & 33.2 & 33.3 & 33.5 & 0.1 & 0.3 & 0.0 \\
\hline $\begin{array}{l}\text { Read } \\
\text { newspapers } \\
\text { almost daily }\end{array}$ & 32.3 & 34.3 & 33.3 & 0.1 & 0.2 & 0.0 \\
\hline $\begin{array}{l}\text { Ever had } \\
\text { premarital sex }\end{array}$ & $20.4^{b}$ & 11.1 & 11.4 & $3.4^{\mathrm{b}}$ & 10.4 & 8.0 \\
\hline $\begin{array}{l}\text { Ever used } \\
\text { drugs }\end{array}$ & $2.0^{b}$ & 0.5 & 0.4 & 0.5 & 1.9 & 0.4 \\
\hline $\begin{array}{l}\text { Ever had sex } \\
\text { with a sex } \\
\text { worker }\end{array}$ & 4.3 & 5.5 & 2.3 & 0.1 & 2.2 & 0.9 \\
\hline
\end{tabular}

ACASI: Audio computer-assisted self interview; SA: Self-administered paper-andpencil interview; PI: Personal interview

a Both sexes combined, since differences between them were nonsignificant

${ }^{\mathrm{b}} \mathrm{p}<0.05$ ACASI vs. other two methods

SA and $6.4 \%$ in PI, respectively). The ACASI group also showed significantly higher $(p<0.05)$ affirmative response rates than the other two groups in both rural and urban settings. In urban districts, $22.3 \%$ of ACASI respondents reported having had premarital sex (versus $13.7 \%$ and $11.3 \%$ of SA and PI respondents, respectively). In rural areas, percentages in ACASI, SA and PI groups were $19 \%, 8.6 \%$ and $11.4 \%$ respectively (Figure 1).

Figure 1: Interview mode and mean number of sexual partners in previous 12 months by marital status, sex and rural/urban locality

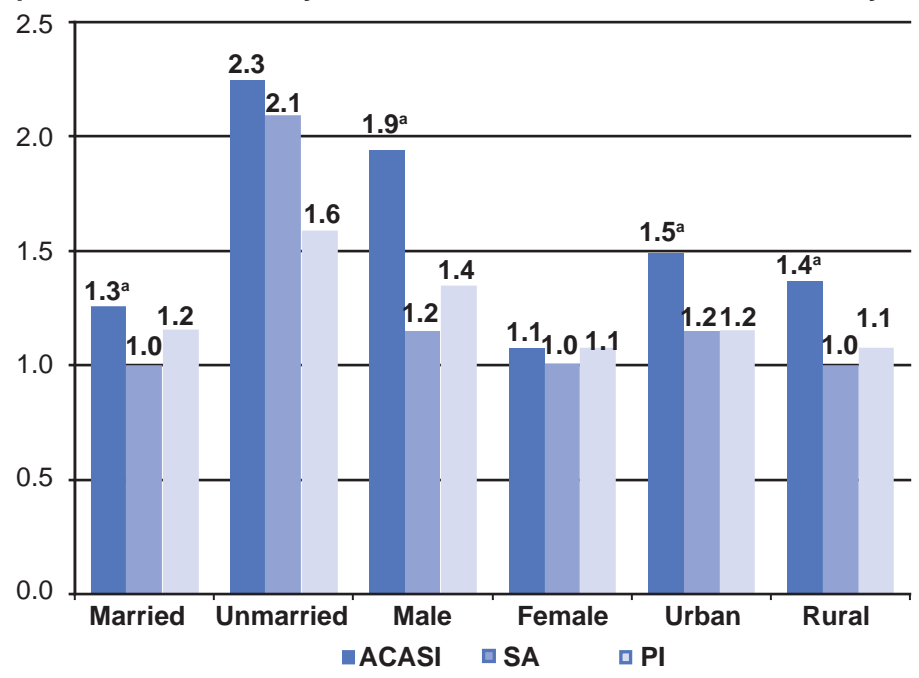

${ }^{a} p<0.05$ ACASI vs. other two methods

Sexual behavior Respondents in the three groups were compared by mean reported number of sexual partners over the previous 12 months and mean number of lifetime sexual partners (among the sexually active sample) in different subgroups (married and unmarried; men and women; urban and rural). Mean number of lifetime partners was similar in all subgroups, but mean number of reported sexual partners in the previous 12 months in the married ACASI group was 1.34 (95\% Cl 1.21-1.47) versus 1.04 (95\% Cl 1.02-1.06) in SA and 1.16 (95\% Cl 1.10-1.21) in the PI group, respectively. Figure 1 shows the breakdown for the latter question by sex, marital status and urban/rural locality. In
Table 4: Multinominal logistic regression model for affirmative response (Yes) and refusal by sexually active Vietnamese men to question Have you ever had sex with a sex worker? $(\mathrm{N}=1452)$

\begin{tabular}{|c|c|c|}
\hline \multirow{3}{*}{ Variable } & \multicolumn{2}{|l|}{ Response } \\
\hline & Yes & Refusal \\
\hline & OR $(95 \% \mathrm{Cl})$ & OR $(95 \% \mathrm{Cl})$ \\
\hline \multicolumn{3}{|l|}{ Socioeconomic status } \\
\hline Lowest vs. highest quintile & $0.5(0.2-1.3)$ & $1.1(0.6-2.0)$ \\
\hline Second lowest vs. highest quintile & $0.5(0.2-1.2)$ & $0.8(0.5-1.5)$ \\
\hline Middle vs. highest quintile & $0.8(0.4-1.7)$ & $0.9(0.5-1.6)$ \\
\hline Second highest vs. highest quintile & $0.6(0.3-1.3)$ & $0.6(0.4-1.1)$ \\
\hline \multicolumn{3}{|l|}{ Marital status } \\
\hline Single vs. ever married & $1.4(0.6-3.1)$ & $50.4(32.9-77.1)$ \\
\hline \multicolumn{3}{|l|}{ Age group (years) } \\
\hline $15-19$ vs. $40-49$ & $1.1(0.3-4.6)$ & $6.4(3.3-12.5)$ \\
\hline $20-39$ vs. $40-49$ & $0.9(0.5-1.6)$ & $1.0(0.6-1.5)$ \\
\hline \multicolumn{3}{|l|}{ Interview method } \\
\hline ACASI vs. PI & $2.8(1.4-5.6)^{a}$ & $0.3(0.2-0.5)^{b}$ \\
\hline SA vs. PI & $2.3(1.2-4.6)^{a}$ & $1.4(0.9-2.1)$ \\
\hline \multicolumn{3}{|l|}{ Location } \\
\hline Ha Noi vs. Can Tho & $1.0(0.5-1.8)$ & $1.6(1.0-2.4)$ \\
\hline Da Nang vs. CanTho & $0.6(0.3-1.2)$ & $1.8(1.2-2.8)^{2}$ \\
\hline \multicolumn{3}{|l|}{ Rural/Urban } \\
\hline Rural vs. urban & $1.9(1.1-3.4)^{\mathrm{a}}$ & $1.9(1.3-2.7)^{a}$ \\
\hline
\end{tabular}

ap $<0.05$

${ }^{\mathrm{b} p}<0.01$

the married sample, the mean number of sexual partners reported by the ACASI group was significantly higher than reported in the other two groups; the same held true for both the urban and rural sample, but not for unmarried respondents nor for women.

A multinominal logistic regression model was constructed to predict likelihood of reporting having sex with sex workers among the sexually active male sample (Table 4). Adjusting for marital status, age, rural/urban, city and socioeconomic variables included in the model, a male subject in the ACASI group was 2.8 times more likely to answer "yes" to the question "Have you ever had sex with a sex worker?" than was a male subject in the PI group (OR 2.8, $\mathrm{Cl}$ 1.4-5.6). Male subjects in the SA group were also more likely to answer "yes" to this question compared to their PI counterparts (OR 2.3, Cl 1.2-4.6). Probability of refusal was much lower in the ACASI group than in the interview group (OR 0.3, $\mathrm{Cl} 0.2-0.5$ ).

The multinominal model also revealed marital status, age and city of residence to be related to sexually active men's probability of refusal to answer the same question, with odds as follows: unmarried men, OR 50.4 ( $\mathrm{Cl}$ 32.9-77.1); men aged 15 to 19 years versus men aged 40 to 49 years, OR 6.4 ( $\mathrm{Cl} 3.3-12.5)$; men in Da Nang versus men in Can Tho, OR 1.8 (Cl 1.2-2.8); rural men versus urban, OR 1.9 (Cl 1.3-2.7).

\section{DISCUSSION}

This survey aimed to extract lessons and insights for a later broader study. Previous research worldwide and in Vietnam found rates of successful interviews higher using ACASI than either PI or SA.[4,10,17] Le et al. also reported that response rates were higher in younger respondents when ACASI was applied. In 2001, Bui's population survey in Quang Ninh using a tape-recorded questionnaire elicited a response rate of $97 \%$ for persons aged 
15 to 45 years.[18] A more recent survey in Hai Phong in 2008 (using a CD player to play prerecorded questions) with a sample aged 18 to 29 years reported a response rate of 98\%.[19] However, other national studies in young populations using non-ACASI methods-SAVY1 and SAVY2-reported interview success rates of only $85 \%$.[20]

The overall success rate of about $88 \%$ in this study was therefore very good. The interview success rate for the ACASI group was $86 \%$, slightly but not significantly lower than the rates for other methods; and ACASI also resulted in similar refusal rates. Given that other variables were distributed similarly in the three interview modes, the difference in proportion of male and female respondents was probably chance. Also, there were no statistically significant differences among the three methods with regard to non-sensitive questions, suggesting that for routine factual questions, ACASI has no particular advantage over SA and PI, consistent with previous findings in Vietnam and elsewhere.[5,11,21]

The fact that the SA sample tended to have the highest refusal rates on sensitive questions raises issues about the applicability of SA compared to the other two methods. It is likely that people felt more comfortable and less "guilty" skipping questions in SA mode than in the other two methods, as there is no pressure for them to fill in the box when they handle the forms on their own. In contrast, a clear pattern emerged revealing that when asked by ACASI, respondents are less likely to refuse. Responses to selected questions at different levels of sensitivity are intriguing in that the ACASI group showed slightly lower affirmative response rates compared to $\mathrm{PI}$ in most non-sensitive topics, but higher rates in sensitive ones. Responses for sensitive issues did not differ between urban and rural settings; although rates of reported premarital sex were slightly higher in urban respondents than in rural. They were at least eight percentage points higher in the ACASI group in both settings. No previous study in Vietnam was able to look at this technique in rural samples,[10] so this finding is helpful and supports the idea of using ACASI in both rural and urban regions in future research.

Drug use is a highly sensitive behavioral question and in this respect, our findings revealed a clear advantage of ACASI, with consistently higher affirmative response rates than SA and PI for both sexes and all locations. Percentages of reported premarital sexual relationships were also significantly higher in the ACASI group (20.4\%); the same trend has been reported previously but the difference found here-almost twofold-was even greater than seen in previous studies.[10]

A similar pattern was found regarding sexual relationships. Interestingly, as hypothesized, male subjects consistently showed a trend to greater response differences with ACASI than their female counterparts. However, due to small sample size in the female sexually active group, only the mean number of men's sexual partners in the last 12 months was found significantly higher in the ACASI group. In other words, for men, ACASI seems to be more appropriate and yield more reliable affirmative responses for such sensitive topics. This is consistent with a previous ACASI trial in Vietnam, which found similar high response rates on sensitive questions about sexual risk behaviors among male youth in Vietnam, but lower response rates in young women.[10] This pattern is, however, opposite to that found in several studies in Africa, in which young women showed higher responsiveness to ACASI.[5,6,22] Other factors, such as differing levels of comfort with technology, could also contribute to the apparent lack of advantage for female respondents seen for ACASI in this study. Determining whether it is an artifact of study power or a true difference would require a study with larger sample size for women.

The findings from the multinominal logistic regression model again affirmed the advantage of using ACASI compared to SA and PI methods in the specific sensitive question of having sex with sex workers. Data also suggest that those in the SA group were significantly more likely to "skip" such tough questions. This could be a threat to the validity of the findings in the SA group, where the refusal rate is much higher than in the ACASI and PI groups. The other significant results for independent variables in the multinomial model were also culturally understandable, as male youth-especially if married-may be less comfortable than more senior men speaking openly about such a risk behavior. Also, social norms and pressures in more urbanized settings may make men more likely to choose the option appearing more neutral and less provocative, i.e., selecting the "no information response". This may help to explain why men in $\mathrm{Ha}$ Noi, Da Nang (both more urbanized than Can Tho) were more likely to refuse than their counterparts in Can Tho. However, no previous study in Vietnam has reported on this issue, so we have no basis for comparison.

There are a number of limitations in a study of this kind. First, the sample is drawn from only three cities, with no pretensions to national representation. Thus, the findings cannot be generalized to Vietnam as a whole. Second, low prevalence of some sexual behaviors limits possibilities both for comparison and data disaggregation, such as between married and unmarried samples. Third, the study was not able to differentiate honesty from accuracy, although certainly one could argue that honesty is more likely to produce greater accuracy than dishonesty.

In any case, there is always the possibility that some respondents-especially young men-may have exaggerated their sexual life. Or, on the other hand, some respondents may still have been sufficiently uncomfortable in the home setting, and thus under-reported high-risk behaviors. These issues have been discussed by other researchers previously as a source of information bias, regardless of interview method.[23]

Another limitation may be low literacy and lack of familiarity with a computer keyboard. Such issues have been reported and discussed in feasibility studies of ACASI in countries such as Peru, China, India and Russia.[24] In this study, however, we purposely selected three cities with rather high literacy levels. More importantly, low refusal rates in ACASI respondents support the idea that ACASI was not too difficult for this sample. Some authors have suggested that a more user-friendly version of ACASI might better facilitate its use, irrespective of educational level.[23] Another previous study in adolescents in Ha Noi used an additional keypad attached to the laptop with a colored sticker for answer entry, allowing illiterate respondents to answer more easily.[10] However, such adaptations are more costly and complicated.

Although this is not a limitation of the study per se, one might argue that costs may present a barrier for wider use of ACASI. It is certainly an issue that research teams have to consider carefully, but in our experience, use of laptops for interviews can offset other costs. For example, data entry costs are reduced because respon- 
dents directly key in their answers. Also, laptop prices have been decreasing rapidly, and we can certainly use these laptops (and installed software) for similar projects in the future, at least for a few more years. Therefore, despite some limitations, we consider ACASI a promising alternative in studies to assess risk behavior in Vietnam. Further studies targeting male respondents in the area of HIVIAIDS (such as surveys of men who have sex with men) should consider this application to obtain more honest answers, which we consider more likely to produce accurate information.

\section{CONCLUSIONS}

Program implementers in HIV-related projects as well as researchers and policymakers have had a legitimate concern that sensitive issues such as unsafe sexual practices and other risk behaviors tend to be under-reported in surveys and assessments, leading to development of interventions based on skewed and faulty infor- mation. This study confirmed the applicability and advantages of ACASI compared to traditional interview modes in improving item response rates and responses to sensitive questions, especially in male respondents (in both rural and urban settings). Although ACASI still has not been demonstrated to have advantages for use with female respondents in the Vietnamese context, it is a promising methodology that may help increase honesty, leading to greater accuracy of future data collection on sensitive topics.

\section{ACKNOWLEDGMENTS}

This study was supported by a PEPFAR grant to the Hanoi School of Public Health under the project Partnering with Hanoi School of Public Health to Enhance Public Health Capacity for HIV Prevention and Care Activities in Vietnam. The authors would like to thank the Hanoi School of Public Health and PEPFAR/CDC Vietnam for their support. -1 -

\section{REFERENCES}

1. Vietnam 2010 Country Progress Report on HIV/ AIDS. Ha Noi: Ministry of Health (VN); 2010 Mar.

2. Thuong NV, Nhung VT, Nghia KV, Tram LT, O'Farrell N. HIV in female sex workers in five border provinces of Vietnam. Sex Transm Infect. 2005 Dec;81(6):477-9.

3. General Statistical Office (VN), National Institute of Hygiene and Epidemiology (VN), ORC Macro. Vietnam Population and AIDS Indicator Survey 2005. Calverton, Maryland (US): ORC Macro; 2006 May.

4. Metzger DS, Koblin B, Turner C, Navaline H, Valenti $\mathrm{F}$, Holte $\mathrm{S}$, et al. Randomized controlled trial of audio computer-assisted self-interviewing: utility and acceptability in longitudinal studies. Am J Epidemiol. 2000 Jul 15;152(2):99-106.

5. Mensch BS, Hewett PC, Erulkar AS. The reporting of sensitive behavior by adolescents: a methodological experiment in Kenya. Demography. 2003 May; 40(2):247-68.

6. Mensch BS, Hewett PC, Gregory R, Helleringer S. Sexual behavior and STI/HIV status among adolescents in rural Malawi: an evaluation of the effect of interview mode on reporting. Stud Fam Plann. 2008 Dec;39(4):321-34.

7. Gribble JN, Miller HG, Rogers SM, Turner CF. Interview mode and measurement of sexual behaviors: Methodological issues. J Sex Res. 1999 Feb;36(1):16-24.

8. Estes LJ, Lloyd LE, Teti M, Raja S, Bowleg L, Allgood KL, et al. Perceptions of audio computerassisted self-interviewing (ACASI) among women in an HIV-positive prevention program. PLoS One. 2010 Feb 10;5(2):e9149.

9. Phillips AE, Gomez GB, Boily MC, Garnett GP. A systematic review and meta-analysis of quantitative interviewing tools to investigate self-reported HIV and STI associated behaviours in low- and middle-income countries. Int J Epidemiol. 2010 Dec;39(6):1541-55.

10. Le LC, Blum RW, Magnani R, Hewett PC, Do HM. A pilot of audio computer-assisted self-interview for youth reproductive health research in Vietnam. J Adolesc Health. 2006 Jun;38(6):740-7.

11. Le Linh C, Blum RW. Premarital sex and condom use among never married youth in Vietnam. Int J Adolesc Med Health. 2009 JulSep;21(3):299-312.

12. Warner SL. Randomized response: A survey technique for eliminating evasive answer bias. J Am Stat Assoc. 1965 Mar;60(309):63-6.

13. Tamhane AC. Randomized response techniques for multiple sensitive attributes. J Am Stat Assoc. 1981 Dec;76(376):916-23.

14. Chaudhuri A, Mukherjee R. Randomized Response Techniques: A Review. Statistica Neerlandica. 1987 Mar;41(1):27-44.

15. Lensvelt-Mulders GJLM, Hox JJ, van der Heijden PGM, Maas CJM. Meta-Analysis of Randomized Response Research. Sociol Methods Res. 2005;33(3):319-48.

16. Peeters CFW, Lensvelt-Mulders GJLM, Lasthuizen $\mathrm{K}$. A note on a simple and practical randomized response framework for eliciting sensitive dichotomous and quantitative information. Sociol Methods Res. 2010 Sep;39(2):283-96.

17. Tourangeau R, Smith TW. Asking sensitive questions: The impact of data collection mode, question format and question content. Public Opin Q. 1996;60(2):275-304.

18. Bui TD, Pham CK, Pham TH, Hoang LT, Nguyen TV, Vu TQ, et al. Cross-sectional study of sexual behaviour and knowledge about HIV among urban, rural, and minority residents in Viet Nam. Bull World Health Organ. 2001;79(1):15-21.

19. Duong CT, Nguyen TH, Hoang TT, Nguyen VV, Do TM, Pham VH, et al. Sexual risk and bridging behaviors among young people in Hai Phong, Vietnam. AIDS Behav. 2008 Jul;12(4):643-51.

20. Ministry of Health (VN), GSO, ADB. Survey Assessment of Vietnamese Youth 2 (SAVY2); Ha Noi: General Office of Population and Family Planning, 2010.
21. Des Jarlais DC, Paone D, Milliken J, Turner CF, Miller H, Gribble J, et al. Audio-computer interviewing to measure risk behaviour for HIV among injecting drug users: a quasi-randomised trial. Lancet. 1999 May 15;353(9165):1657-61.

22. Glynn JR, Kayuni N, Banda E, Parrott F, Floyd $\mathrm{S}$, Francis-Chizororo $\mathrm{M}$, et al. Assessing the validity of sexual behaviour reports in a whole population survey in rural malawi. PLoS One. 2011;6(7):e22840.

23. van der Elst EM, Okuku HS, Nakamya $P$, Muhaari A, Davies A, McClelland RS, et al. Is Audio Computer-Assisted Self-Interview (ACASI) Useful in Risk Behaviour Assessment of Female and Male Sex Workers, Mombasa, Kenya? PLoS ONE. 2009;4(5):e5340.

24. NIMH Collaborative HIVISTD Prevention Trial Group. The feasibility of audio computer-assisted self-interviewing in international settings. AIDS. 2007 Apr;21 Suppl 2:S49-58.

\section{THE AUTHORS}

Linh Cu Le (Corresponding author: Icl@hsph. edu.vn), physician with a master's degree in applied development and a doctorate in public health. Chair, department of demography, Hanoi School of Public Health, Vietnam.

Lan T.H. Vu, physician with a doctorate in epidemiology. Chair, department of epidemiology and biostatistics, Hanoi School of Public Health, Vietnam.

Submitted: December 31, 2011

Approved for publication: August 22, 2012

Disclosures: None 